\title{
Prognostic and clinicopathological significance of platelet to lymphocyte ratio in esophageal cancer: a meta-analysis
}

\author{
Juhong Deng', Peng Zhang', Yue Sun², Ping Peng', Yu Huang ${ }^{2}$ \\ ${ }^{1}$ Department of Endocrinology, Liyuan Hospital, ${ }^{2}$ Department of Oncology, Tongji Hospital, Tongji Medical College, Huazhong University of \\ Science and Technology, Wuhan 430030, China \\ Contributions: (I) Conception and design: Y Huang; (II) Administrative support: Y Sun, P Peng; (III) Provision of study materials or patients: Y \\ Huang, J Deng; (IV) Collection and assembly of data: Y Sun, J Deng, P Zhang; (V) Data analysis and interpretation: Y Huang, J Deng, P Zhang; (VI) \\ Manuscript writing: All authors; (VII) Final approval of manuscript: All authors. \\ Correspondence to: Yu Huang. Department of Oncology, Tongji Hospital, Tongji Medical School, Huazhong University of Science and Technology, \\ No.1095 Jie Fang Avenue, Hankou, Wuhan 430030, China. Email: huangyu03tj@163.com.
}

Background: The prognostic and clinicopathological significance of the platelet to lymphocyte ratio (PLR) has been studied in various cancers. However, studies examining the role of PLR in esophageal cancer have not yielded consistent results. The purpose of this meta-analysis was to study the prognostic and clinicopathological significance of PLR in esophageal cancer patients.

Methods: We performed a literature search in three major databases: PubMed, Web of Science and Embase (up until May 1, 2017). The clinicopathologic significance of PLR and its prognostic significance were analyzed.

Results: Our meta-analysis consisted of 13 studies with 4,621 patients. The pooled hazard ratios (HRs) showed that a high PLR was associated with poor survival of esophageal cancer $[H R=1.283 ; 95 \%$ confidence interval (CI): 1.173-1.404; $\mathrm{P}<0.001$ ]. Subgroup analysis revealed that elevated PLR was associated with poor survival in esophageal squamous cell carcinoma ( $\mathrm{HR}=1.281 ; 95 \% \mathrm{CI}: 1.098-1.493 ; \mathrm{P}=0.002)$. The pooled odds ratio (OR) indicated that high PLR was also associated with the depth of tumor invasion (OR $=1.543$, 95\% CI: 1.269-1.876, $\mathrm{P}<0.001$ ), lymph node metastasis (OR =1.427, 95\% CI: $1.195-1.705, \mathrm{P}<0.001)$, tumor length $(\mathrm{OR}=1.81,95 \% \mathrm{CI}: 1.331-2.461, \mathrm{P}<0.001)$, and Tumor stage (OR =1.459, 95\% CI: 1.235-1.724, $\mathrm{P}<0.001)$.

Conclusions: Our results demonstrate that elevated PLR was significantly associated with poor prognosis of esophageal cancer. Furthermore, the high PLR might predict worse clinicopathological features of esophageal cancer patients.

Keywords: Meta-analysis; esophageal neoplasms; platelet to lymphocyte ratio (PLR); prognosis

Submitted Sep 09, 2017. Accepted for publication Jan 26, 2018.

doi: $10.21037 /$ jtd.2018.02.58

View this article at: http://dx.doi.org/10.21037/jtd.2018.02.58

\section{Introduction}

Esophageal cancer is one of the most common cancer types worldwide with a poor prognosis. Although multimodal therapeutic strategies have been used to treat esophageal cancer, including radical operation, chemotherapy, and radiotherapy, the 5-year survival rate remains low (1-3). The identification of prognostic factors in esophageal cancer is thus urgently required to better treat this disease.

Previous studies have established a close relationship between inflammation and cancer (4). Chronic inflammation can induce the development of various types of cancers, and the microenvironment caused by inflammation or by the oncogenic changes during tumorigenesis may promote cancer angiogenesis and metastasis $(4,5)$. Some inflammatory factors in the blood, such as lymphocytes, neutrophils, 
monocytes and platelets, show alterations in cancer (6-8). Many inflammatory factors have been associated with prognosis in various cancers. The combination of inflammatory factors, such as platelet-to-lymphocyte ratio (PLR), was also reported to be a prognostic factor in many cancers such as breast, lung and gastric cancers (9-12).

Recent studies have shown that a high PLR might be associated with poor prognosis of esophageal cancer $(13,14)$. However, this result was not confirmed in other studies $(15,16)$. In addition, several inflammatory factors may affect tumor characteristics, such as tumor length and depth of tumor invasion. We performed this meta-analysis to examine the potential role of PLR in the prognosis of esophageal cancer and its relationship with tumor pathological characteristics.

\section{Methods}

\section{Search strategy}

We performed a literature search in the PubMed, Embase, and Web of Science databases (up until May 1, 2017). We used the following search terms: prognosis (e.g., "prognoses", "prognostic", "survival”), PLR (e.g., "platelet lymphocyte ratio", "platelet to lymphocyte ratio", "plateletto-lymphocyte ratio"), and esophageal cancer (e.g., "esophageal neoplasm", "esophageal cancer", "esophageal carcinoma") (details seen in Supplementary).

\section{Selection criteria}

We used the following criteria for inclusion: the diagnosis for esophageal cancer was proven by pathology, and the correlation between pretreatment PLR and overall survival (OS) was studied. The exclusion criteria were as follows: article types of reviews, letters, abstracts, and case reports; studies written in a language other than English; absence of hazard ratio (HR) and $95 \%$ confidence interval (CI) values; and studies without a cutoff value of PLR.

\section{Data extraction and quality assessment}

Two researchers (Deng JH and Sun Y) independently extracted data from relevant studies. In cases of disagreements, another researcher (Zhang P) was consulted. The following information was collected: authors' names, year of publication (we included the final published year), study country, study design, gender, age, stage of disease, pathological type, cutoff values for PLR, HR with 95\% CI of PLR for OS, type of study, treatment intent, treatment strategy and survival data. We used the Newcastle-Ottawa Scale (NOS) for quality assessment of the included research (http://www.ohri.ca/programs/clinical_epidemiology/ oxford.asp). If the NOS score was $\geq 6$ points, we considered the article was high quality

\section{Statistical analysis}

Meta-analysis was performed using Stata 12.0 software (STATA, College Station, TX, USA). HRs for the association of PLR and esophageal cancer were obtained from the research. When HRs was reported in both univariate and multivariate analyses, we used the multivariate-adjusted HRs. The pooled HRs was finally summarized from each study of HRs and their 95\% CI. The I-squared $\left(\mathrm{I}^{2}\right)$ statistic was used to evaluate the heterogeneity of the studies. If $\mathrm{I}^{2}$ $>50 \%$, meta-analysis was chosen to evaluate with a random effects model; otherwise, the fixed effect model was chosen. Odds ratios (ORs) were used to estimate the association between PLR and clinicopathological characteristics. We estimated publication bias by Begg's funnel plot test and Egger's linear regression test. We also used country, cutoff, patient's number (simple size), treatment strategy and pathologic type for subgroup analysis.

\section{Results}

\section{Study Characteristics}

A total of 59 studies were initially identified after searching the databases. After browsing the title and abstracts, 34 studies were excluded. We then reviewed the remaining 25 studies and excluded 12 studies for the following reasons: two studies used data with a continuous PLR level for survival analysis, without a clear cutoff value; one study performed the analysis in the samples with a repeated population; four studies did not use OS as an endpoint; two studies did not describe the relationship between PLR and OS; and three studies did not report the HR value. A final 13 studies were included in the meta-analysis, with 4,621 patients (Figure 1) (13-25). These studies were published from 2013 to 2017. The most common type of pathology involved in these studies was esophageal squamous carcinoma (ESCC). The PLR cutoff point ranged from 117.07 to 244 . The detailed information of these studies is shown in Table 1. NOS scores of all the studies were at least 6 or more. 


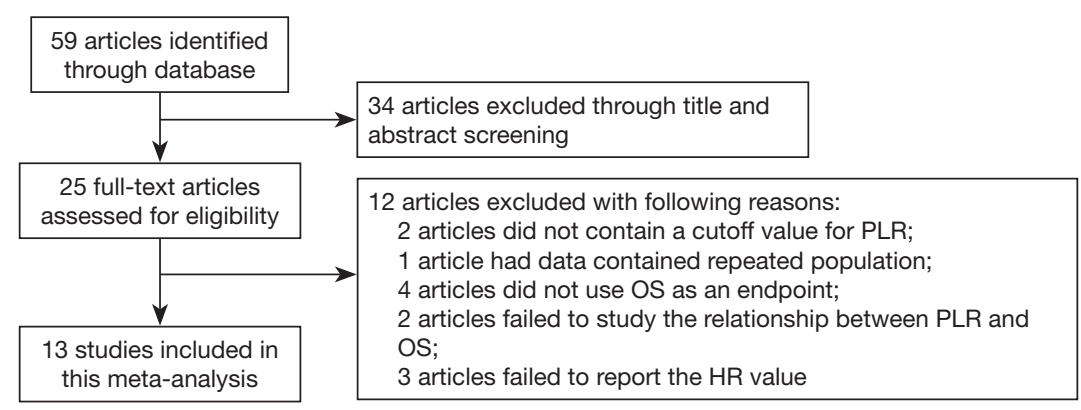

Figure 1 The flow diagram for selection of studies. PLR, platelet to lymphocyte ratio; OS, overall survival; HR, hazard ratio.

\section{The role of PLR in the prognosis of esophageal cancer}

All 13 studies retrospectively reported the relationship between PLR and OS. We found that a high PLR was positively related to poor OS (pooled $\mathrm{HR}=1.283,95 \%$ CI: 1.173-1.404; $\mathrm{P}<0.001$, Figure 2). We used the fixedeffect model, for no obvious heterogeneity existed $\left(\mathrm{I}^{2}=49.4 \%\right.$, $\mathrm{P}_{\mathrm{h}}=0.022$ ). First we performed subgroup analysis with the pathologic type. We found that 10 studies reported the association between PLR and OS in ESCC and demonstrated that a high PLR was related to poor OS ( $\mathrm{HR}=1.281,95 \%$ CI: $1.098-1.493, \mathrm{P}=0.002)$ of ESCC (Figure 3); here we used random effect model for $\mathrm{I}^{2}>50 \%$. We also performed subgroup analysis by cutoff value, sample size and countries. Subgroup analysis by cutoff values showed an HR for cutoff value $\geq 150$ of 1.413 (95\% CI: $\left.1.136-1.758, \mathrm{P}=0.002, \mathrm{I}^{2}=56.1 \%, \mathrm{P}_{\mathrm{h}}=0.026\right)$ and for cutoff value $<150$ of 1.187 (95\% CI: $1.05-1.341 ; \mathrm{P}=0.006$; $\left.\mathrm{I}^{2}=7.4 \%, \mathrm{P}_{\mathrm{h}}=0.365\right)$. In patients treated with surgery alone, the combined HR was 1.407 (95\% CI: 1.018-1.945; $\left.\mathrm{P}=0.039 ; \mathrm{I}^{2}=73.2 \%, \mathrm{P}_{\mathrm{h}}=0.024\right)$; for research with multimodal treatment, the HR was 1.255 (95\% CI: $1.102-1.43$; $\mathrm{P}=0.001 ; \mathrm{I}^{2}=48.6 \%, \mathrm{P}_{\mathrm{h}}=0.049$ ) (Table 2). Subgroup analyses by sample size and countries are shown in Table 2 .

\section{PLR and clinicopathological features}

The association between PLR and clinicopathological characteristic features was studied in several articles (1315,18,21-25). Six studies reported the association of PLR with depth of tumor invasion in esophageal cancer. The combined OR revealed that a high PLR was related to a deeper tumor invasion (OR $=1.543$, 95\% CI: $1.269-1.876$, $\mathrm{P}<0.001)$. Seven studies reported that a high PLR was positively associated with lymph node metastasis of esophageal cancer $(\mathrm{OR}=1.427,95 \% \mathrm{CI}: 1.195-1.705$,
$\mathrm{P}<0.001)$. Furthermore, a high PLR was positively associated a longer tumor length (OR $=1.810$; 95\% CI: 1.331-2.461, $\mathrm{P}<0.001)$ and a higher $\mathrm{TNM}$ stage $(\mathrm{OR}=1.459,95 \%$ CI: $1.235-1.724, \mathrm{P}<0.001)$. The level of PLR was not related to other clinicopathological factors (tumor differentiation $\mathrm{OR}=1.196,95 \% \mathrm{CI}$ : 0.978-1.462; $\mathrm{P}=0.081$; vascular invasion $\mathrm{OR}=1.104,95 \% \mathrm{CI}: 0.709-1.717$, $\mathrm{P}=0.663$ ) (Table 3).

\section{Publication bias}

Egger's and Begg's test was used evaluate publication bias. No publication bias was detected $(\mathrm{P}=0.126$ and $\mathrm{P}=0.127$ for Egger's and Begg's tests, respectively; Figure 4).

\section{Discussion}

Inflammation plays an important role in the development of cancer. The cells and mediators of inflammation create a specific microenvironment, which influences tumor growth, progression, angiogenesis and metastasis (26). Systematic inflammatory response is a highlight in various research studies, and current interest is focused on the prevention and treatment of cancer by addressing abnormal inflammation (27).

Platelets play an important role in immune and inflammation responses and participate in cancer invasion and metastasis (28). Many platelet-associated chemokines can modulate inflammation within the tumor environment and tumor angiogenesis, such as platelet factor 4 (PF4/CXCL4) and connective tissue activating peptide III (CTAP-III) (29). Lymphocytes have been shown to exert an important role in the cancer immunosurveillance process. Shankaran et al. reported that lymphocytes and IFNgamma collaborate to protect against the development of 


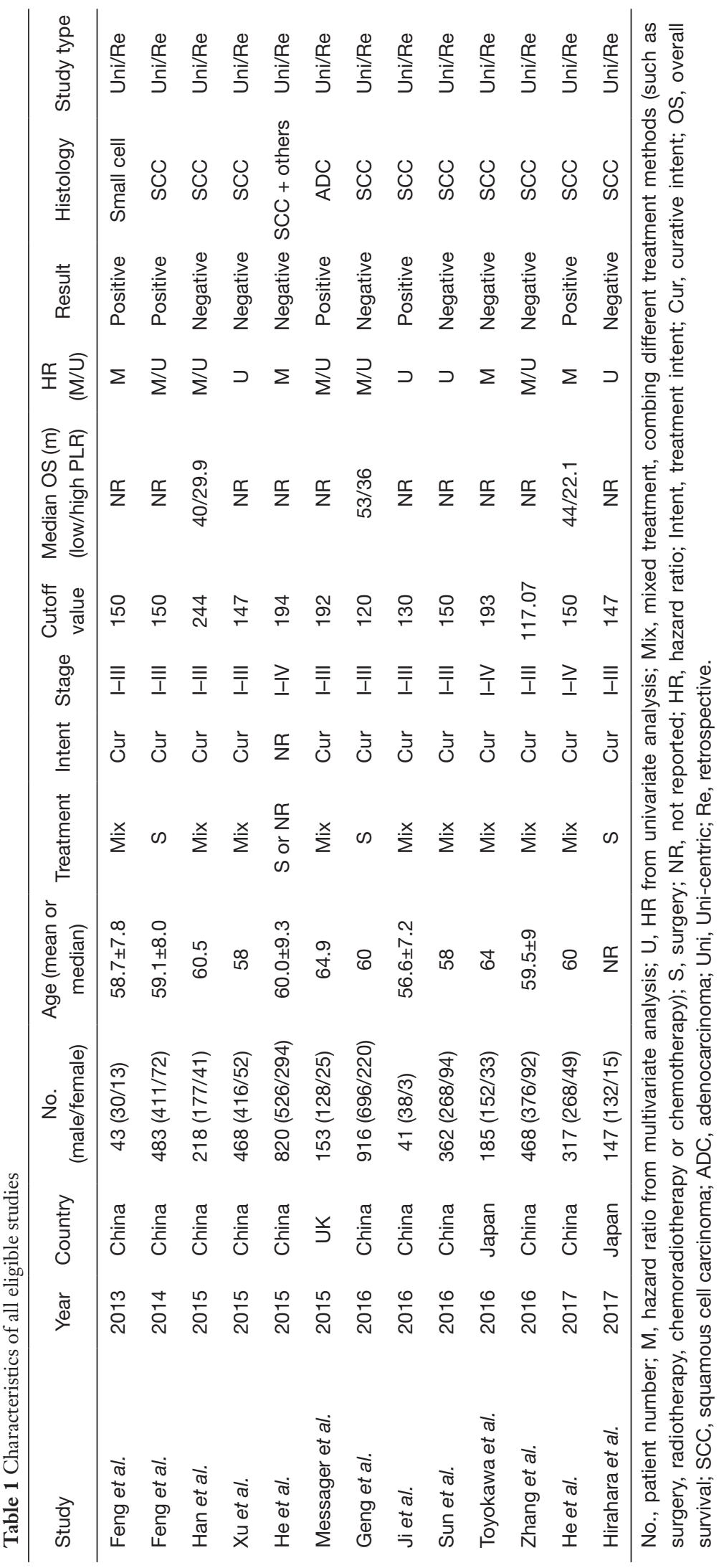




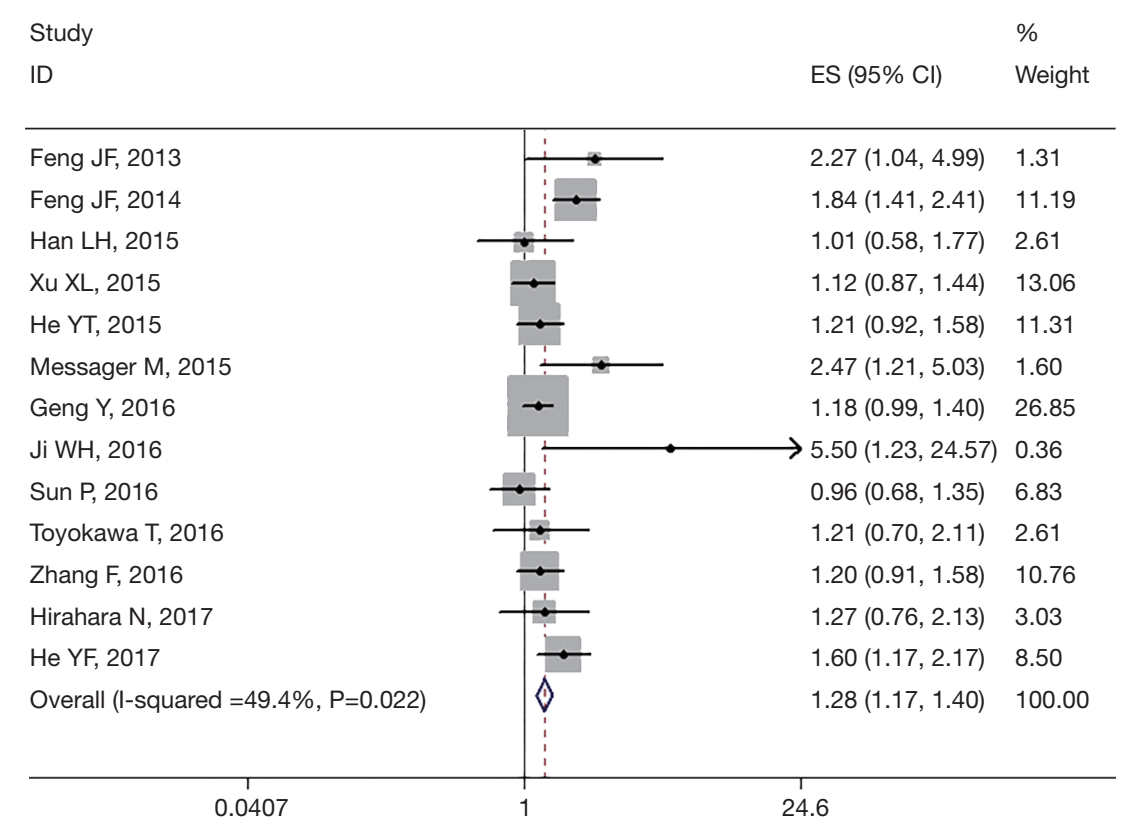

Figure 2 Forest plot of meta-analysis between PLR and OS in esophageal cancer. PLR, platelet to lymphocyte ratio; OS, overall survival; HR, hazard ratio; CI, confidence interval; ES, effect size.

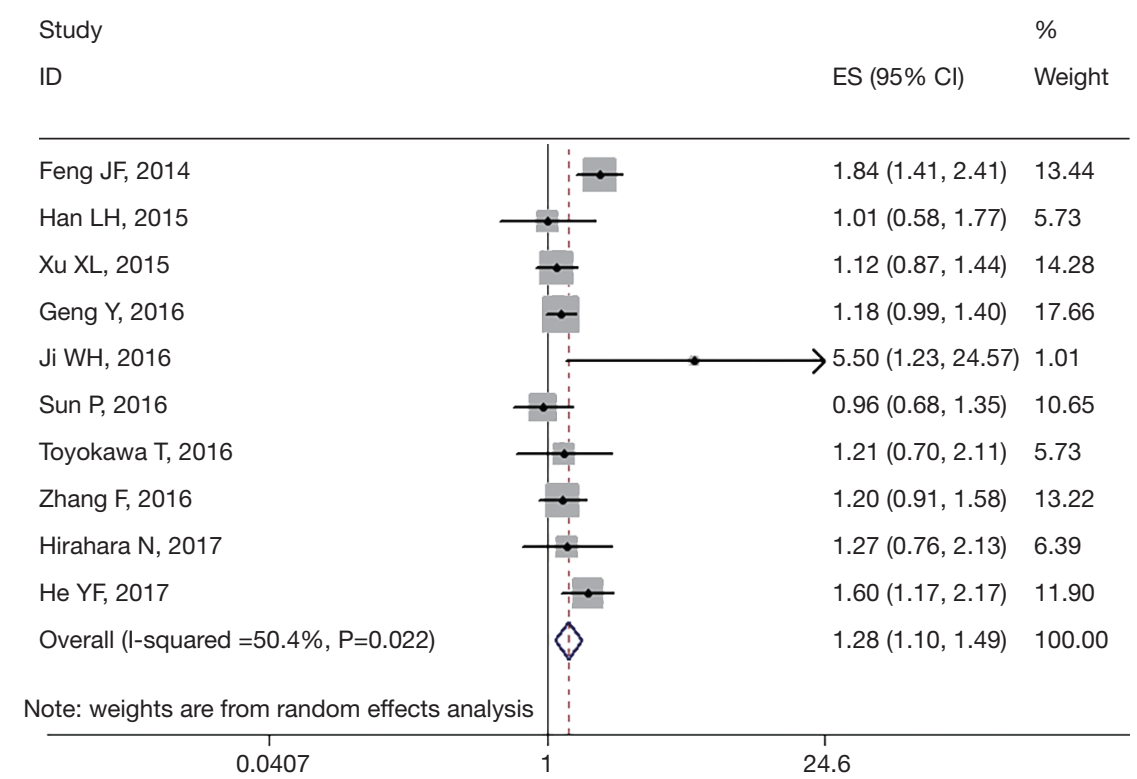

Figure 3 Forest plot of meta-analysis between PLR and OS in ESCC. PLR, platelet to lymphocyte ratio; OS, overall survival; ESCC, esophageal squamous cell carcinoma; HR, hazard ratio; CI, confidence interval; ES, effect size.

chemically-induced sarcomas and spontaneous epithelia carcinomas (30). Inflammation plays an important role in the development of esophageal cancer. Chronic inflammation triggered by environmental exposures may activate the proinflammatory signaling pathway, which promotes the proliferation and survival of cancer cells (31).

The levels of platelets and lymphocytes are altered in the blood of cancer patients $(7,8,32,33)$. Furthermore, the prognostic role of a combination of blood platelets and lymphocytes has been reported in various solid tumors 
Table 2 Meta-analysis results of PLR in esophageal cancer prognosis

\begin{tabular}{|c|c|c|c|c|c|c|c|}
\hline Factor & No. of study & No. of patients & Effect model & HR (95\% Cl) & $\mathrm{P}$ value & \multicolumn{2}{|c|}{ Heterogeneity } \\
\hline \multicolumn{8}{|l|}{ OS } \\
\hline \multirow[t]{2}{*}{ Overall } & 13 & 4,621 & Fix & $1.283(1.173,1.404)$ & 0.000 & 49.4 & 0.022 \\
\hline & & & Random & $1.321(1.146,1.523)$ & 0.000 & & \\
\hline \multirow[t]{2}{*}{ China } & 10 & 4,136 & Fix & $1.271(1.158,1.396)$ & 0.000 & 55.8 & 0.016 \\
\hline & & & Random & $1.300(1.113,1.520)$ & 0.001 & & \\
\hline \multirow[t]{2}{*}{ Japan } & 2 & 332 & Fix & $1.243(0.852,1.814)$ & 0.260 & 0.0 & 0.907 \\
\hline & & & Random & $1.243(0.852,1.814)$ & 0.260 & & \\
\hline Surgery & & & Random & $1.407(1.018,1.945)$ & 0.039 & & \\
\hline \multirow[t]{2}{*}{ Mix } & 9 & 2,255 & Fix & $1.255(1.102,1.430)$ & 0.001 & 48.6 & 0.049 \\
\hline & & & Random & $1.317(1.073,1.615)$ & 0.008 & & \\
\hline \multicolumn{8}{|l|}{ Cutoff value } \\
\hline \multirow[t]{2}{*}{$\geq 150$} & 8 & 2,581 & Fix & $1.407(1.233,1.606)$ & 0.000 & 56.1 & 0.026 \\
\hline & & & Random & $1.413(1.136,1.758)$ & 0.002 & & \\
\hline \multirow[t]{2}{*}{$<150$} & 5 & 2,040 & Fix & $1.187(1.050,1.341)$ & 0.006 & 7.4 & 0.365 \\
\hline & & & Random & $1.189(1.042,1.356)$ & 0.010 & & \\
\hline \multicolumn{8}{|l|}{ Pathologic type } \\
\hline \multirow[t]{2}{*}{ ESCC } & 10 & 3,605 & Fix & $1.267(1.150,1.396)$ & 0.000 & 50.4 & 0.034 \\
\hline & & & Random & $1.281(1.098,1.493)$ & 0.002 & & \\
\hline \multirow[t]{2}{*}{ Other types* } & 3 & 1,016 & & $1.386(1.093,1.759)$ & 0.007 & 60.7 & 0.078 \\
\hline & & & & $1.717(1.014,2.906)$ & 0.044 & & \\
\hline
\end{tabular}

*, the article included other pathologic types except squamous cell carcinoma or with a mixed type. OS, overall survival; ESCC, esophageal squamous cell carcinoma; HR, hazard ratio; $\mathrm{Cl}$, confidence interval; Mix, mixed treatment, combing different treatment methods, including surgery, radiotherapy, chemoradiotherapy or chemotherapy.

$(10,11)$. Evaluation of the PLR is easy to perform in most patients. Some studies have shown that a high PLR might be associated with the poor prognosis of esophageal cancer, but this association has not been completely confirmed.
Therefore, we performed this meta-analysis to confirm the role of PLR in esophageal cancer.

In this meta-analysis, we found that a high PLR predicts poor prognosis in esophageal cancer. We then 
Table 3 Relationship between PLR and the clinicopathologic features

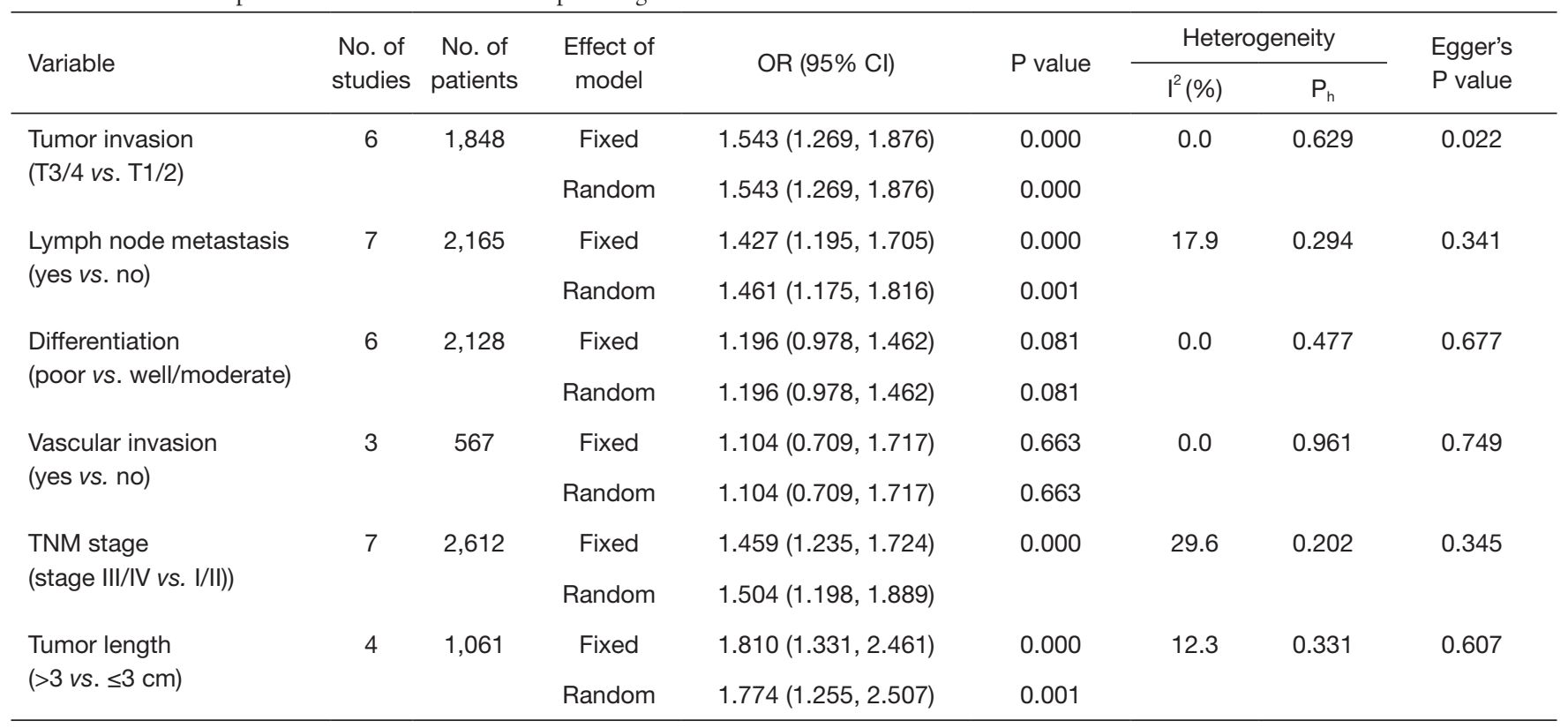

$\mathrm{P}_{\mathrm{h}}, \mathrm{P}$ value for heterogeneity; No., number; OR, odds ratio; Cl, confidence interval; TNM, tumor node metastasis; T, depth of tumor invasion.

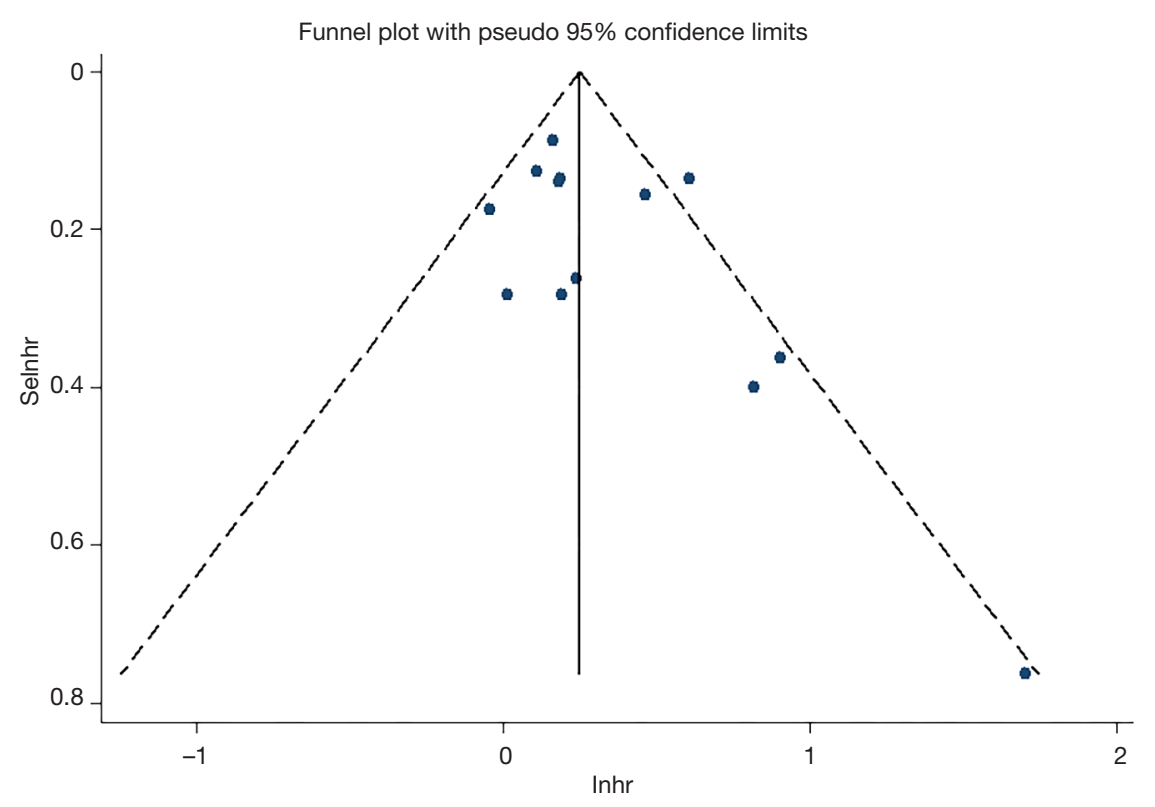

Figure 4 Funnel plot for the publication bias of HR for OS. LNHR, natural logarithm hazard ratio; SELNHR, standard error of natural logarithm hazard ratio; HR, hazard ratio; OS, overall survival.

performed a subgroup analysis and also determined a prognostic role of PLR in ESCC, the major histological type. We also analyzed the relationship between PLR and clinicopathologic features. The result indicated that the elevated PLR level was associated with deeper tumor invasion, lymph node metastasis, longer tumor length and higher tumor stage. Taken together, these data suggest that the level of PLR is important for predicting the prognosis and the status of clinicopathologic features. As well known, the PLR is easily measured in the clinical setting because 
each patient undergoes a blood test before treatment and the cost is low. Thus, PLR might be a useful and convenient tool for clinicians when performing clinical treatment and evaluating the outcomes.

One previous study performed a meta-analysis to investigate the prognostic significance of PLR in esophageal cancer (34). In this previous study, the authors found that high PLR was significantly predictive of poorer OS, deeper tumor invasion, and lymph node metastasis. However, they only included four studies. They did not perform a subgroup analysis according to the pathological types. In the present study, we evaluated a much larger group, including 4,621 patients from 13 studies. We demonstrated for the first time the prognostic role of PLR in ESCC. Furthermore, we concluded that more clinical features might be associated with PLR, such as tumor length and tumor stage.

Our study had several limitations. First, most of the studies were performed in Asian countries (China and Japan), and only one study was performed in a Western country. We did find some studies published in Western countries $(35,36)$, but they did not meet the study criteria. Therefore, our preliminary findings need to be confirmed in other regions. Second, the most prevalent histological type was ESCC. However, different subtypes of esophageal cancer show different biological behaviors and prognoses. Our study did not include enough data on esophageal adenocarcinoma and small cell carcinoma. Only one article in our meta-analysis definitely studied the relationship between esophageal adenocarcinoma and PLR. The researchers found that high PLR is associated with poor OS and disease-free survival (DFS) for esophageal and junction adenocarcinoma (13). This is a positive result but needs further study to prove the result. We should also take this result in caution. Most tumors in their study were located in the esophagus, but a small part of the carcinoma was located in the gastro-esophageal junction, close to the esophagus, often studied, and treated with esophageal tumor (2). In another article, $80 \%$ of the patients were diagnosed as having adenocarcinoma (37). In their study, they found that elevated PLR might be predictive of worse OS in esophageal cancer. However, it was excluded in our meta-analysis for using data without a clear cutoff value for analysis. Therefore, more studies are needed to confirm the role of PLR in other pathological subtypes.

\section{Conclusions}

Our meta-analysis confirmed that a high PLR was associated with poor prognosis of esophageal cancer, especially in ESCC. Furthermore, the PLR level is related to clinicopathologic features of esophageal cancer. More studies are required in other histologic types and geographic regions. More effort is required to predict prognosis of esophageal cancer patients more accurately and develop novel treatment strategies, including anti-inflammatory therapy.

\section{Acknowledgements}

We thank Dr. Xiang Wang for statistic assistance.

Funding: This work was supported by a grant from a research fund of Wuhan Tongii Hospital [2015].

\section{Footnote}

Conflicts of Interest: The authors have no conflicts of interest to declare.

\section{References}

1. Torre LA, Bray F, Siegel RL, et al. Global cancer statistics, 2012. CA Cancer J Clin 2015;65:87-108.

2. Pennathur A, Gibson MK, Jobe BA, et al. Oesophageal carcinoma. Lancet 2013;381:400-12.

3. Sjoquist KM, Burmeister BH, Smithers BM, et al. Survival after neoadjuvant chemotherapy or chemoradiotherapy for resectable oesophageal carcinoma: an updated metaanalysis. Lancet Oncol 2011;12:681-92.

4. Mantovani A, Allavena P, Sica A, et al. Cancer-related inflammation. Nature 2008;454:436-44.

5. Hussain SP, Harris CC. Inflammation and cancer: an ancient link with novel potentials. Int J Cancer 2007;121:2373-80.

6. Kao SC, Pavlakis N, Harvie R, et al. High blood neutrophil-to-lymphocyte ratio is an indicator of poor prognosis in malignant mesothelioma patients undergoing systemic therapy. Clin Cancer Res 2010;16:5805-13.

7. Kobayashi N, Usui S, Kikuchi S, et al. Preoperative lymphocyte count is an independent prognostic factor in node-negative non-small cell lung cancer. Lung Cancer 2012;75:223-7.

8. Kedzierska M, Czernek U, Szydłowska-Pazera K, et al. The changes of blood platelet activation in breast cancer patients before surgery, after surgery, and in various phases of the chemotherapy. Platelets 2013;24:462-8.

9. Gu X, Gao XS, Cui M, et al. Clinicopathological and 
prognostic significance of platelet to lymphocyte ratio in patients with gastric cancer. Oncotarget 2016;7:49878-87.

10. Krenn-Pilko S, Langsenlehner U, Thurner EM, et al. The elevated preoperative platelet-to-lymphocyte ratio predicts poor prognosis in breast cancer patients. Br J Cancer 2014;110:2524-30.

11. Zhao QT, Yuan Z, Zhang H, et al. Prognostic role of platelet to lymphocyte ratio in non-small cell lung cancers: A meta-analysis including 3,720 patients. Int J Cancer 2016;139:164-70.

12. Liu H, Wu Y, Wang Z, et al. Pretreatment platelet-tolymphocyte ratio (PLR) as a predictor of response to firstline platinum-based chemotherapy and prognosis for patients with non-small cell lung cancer. J Thorac Dis 2013;5:783-9.

13. Messager M, Neofytou K, Chaudry MA, et al. Prognostic impact of preoperative platelets to lymphocytes ratio (PLR) on survival for oesophageal and junctional carcinoma treated with neoadjuvant chemotherapy: A retrospective monocentric study on 153 patients. Eur J Surg Oncol 2015;41:1316-23.

14. Feng JF, Huang Y, Zhao Q, et al. Clinical significance of preoperative neutrophil lymphocyte ratio versus platelet lymphocyte ratio in patients with small cell carcinoma of the esophagus. ScientificWorldJournal 2013;2013:504365.

15. Hirahara N, Matsubara T, Kawahara D, et al. Prognostic significance of preoperative inflammatory response biomarkers in patients undergoing curative thoracoscopic esophagectomy for esophageal squamous cell carcinoma. Eur J Surg Oncol 2017;43:493-501.

16. Sun P, Zhang F, Chen C, et al. The ratio of hemoglobin to red cell distribution width as a novel prognostic parameter in esophageal squamous cell carcinoma: a retrospective study from southern China. Oncotarget 2016;7:42650-60.

17. Zhang F, Chen Z, Wang P, et al. Combination of platelet count and mean platelet volume (COP-MPV) predicts postoperative prognosis in both resectable early and advanced stage esophageal squamous cell cancer patients. Tumour Biol 2016;37:9323-31.

18. Yutong H, Xiaoli X, Shumei L, et al. Increased Neutrophil-Lymphocyte Ratio Is a Poor Prognostic Factor in Patients with Esophageal Cancer in a High Incidence Area in China. Arch Med Res 2015;46:557-63.

19. Xu XL, Yu HQ, Hu W, et al. A Novel InflammationBased Prognostic Score, the C-Reactive Protein/Albumin Ratio Predicts the Prognosis of Patients with Operable Esophageal Squamous Cell Carcinoma. PLoS One 2015;10:e138657.
20. Toyokawa T, Kubo N, Tamura T, et al. The pretreatment Controlling Nutritional Status (CONUT) score is an independent prognostic factor in patients with resectable thoracic esophageal squamous cell carcinoma: results from a retrospective study. BMC Cancer 2016;16:722.

21. Ji WH, Jiang YH, Ji YL, et al. Prechemotherapy neutrophil: lymphocyte ratio is superior to the platelet:lymphocyte ratio as a prognostic indicator for locally advanced esophageal squamous cell cancer treated with neoadjuvant chemotherapy. Dis Esophagus 2016;29:403-11.

22. He YF, Luo HQ, Wang W, et al. Preoperative NLR and PLR in the middle or lower ESCC patients with radical operation. Eur J Cancer Care (Engl) 2017;26(2).

23. Han LH, Jia YB, Song QX, et al. Prognostic significance of preoperative lymphocyte-monocyte ratio in patients with resectable esophageal squamous cell carcinoma. Asian Pac J Cancer Prev 2015;16:2245-50.

24. Geng Y, Shao Y, Zhu D, et al. Systemic ImmuneInflammation Index Predicts Prognosis of Patients with Esophageal Squamous Cell Carcinoma: A Propensity Score-matched Analysis. Sci Rep 2016;6:39482.

25. Feng JF, Huang Y, Chen QX. Preoperative platelet lymphocyte ratio (PLR) is superior to neutrophil lymphocyte ratio (NLR) as a predictive factor in patients with esophageal squamous cell carcinoma. World J Surg Oncol 2014;12:58.

26. Candido J, Hagemann T. Cancer-related inflammation. J Clin Immunol 2013;33 Suppl 1:S79-84.

27. Crusz SM, Balkwill FR. Inflammation and cancer: advances and new agents. Nat Rev Clin Oncol 2015;12:584-96.

28. Gresele P, Momi S, Malvestiti M, et al. Platelet-targeted pharmacologic treatments as anti-cancer therapy. Cancer Metastasis Rev 2017;36:331-55.

29. Pilatova K, Greplova K, Demlova R, et al. Role of platelet chemokines, PF-4 and CTAP-III, in cancer biology. J Hematol Oncol 2013;6:42.

30. Shankaran V, Ikeda H, Bruce AT, et al. IFNgamma and lymphocytes prevent primary tumour development and shape tumour immunogenicity. Nature 2001;410:1107-11.

31. Lin EW, Karakasheva TA, Hicks PD, et al. The tumor microenvironment in esophageal cancer. Oncogene 2016;35:5337-49.

32. Zhang J, Huang SH, Li H, et al. Preoperative lymphocyte count is a favorable prognostic factor of disease-free survival in non-small-cell lung cancer. Med Oncol 2013;30:352.

33. Liu P, Zhu Y, Liu L. Elevated pretreatment plasma 
D-dimer levels and platelet counts predict poor prognosis in pancreatic adenocarcinoma. Onco Targets Ther 2015;8:1335-40.

34. Yodying H, Matsuda A, Miyashita M, et al. Prognostic Significance of Neutrophil-to-Lymphocyte Ratio and Platelet-to-Lymphocyte Ratio in Oncologic Outcomes of Esophageal Cancer: A Systematic Review and Metaanalysis. Ann Surg Oncol 2016;23:646-54.

35. Dutta S, Crumley AB, Fullarton GM, et al. Comparison of the prognostic value of tumour- and patient-related factors in patients undergoing potentially curative resection of oesophageal cancer. World J Surg 2011;35:1861-6.

36. Jagadesham VP, Lagarde SM, Immanuel A, et al. Systemic inflammatory markers and outcome in patients with locally advanced adenocarcinoma of the oesophagus and gastrooesophageal junction. Br J Surg 2017;104:401-7.

37. McLaren PJ, Bronson NW, Hart KD, et al. Neutrophilto-Lymphocyte and Platelet-to-Lymphocyte Ratios can Predict Treatment Response to Neoadjuvant Therapy in Esophageal Cancer. J Gastrointest Surg 2017;21:607-13.

Cite this article as: Deng J, Zhang P, Sun Y, Peng P, Huang Y. Prognostic and clinicopathological significance of platelet to lymphocyte ratio in esophageal cancer: a metaanalysis. J Thorac Dis 2018;10(3):1522-1531. doi: 10.21037/ jtd.2018.02.58 


\section{Supplementary}

\section{PubMed search strategy}

((((((((Prognosis) OR Prognoses) OR prognostic) OR outcome) OR survival) OR mortality)) AND $((((($ Esophageal Neoplasm) OR Esophagus Neoplasm) OR Esophagus Cancer) OR Esophageal Cancer) OR esophageal carcinoma) OR Esophagus carcinoma)) AND ((((platelet lymphocyte ratio) OR platelet to lymphocyte ratio) OR platelet-to-lymphocyte ratio) OR PLR)

\begin{tabular}{|c|c|c|}
\hline Search date & Combine & Results \\
\hline \multirow[t]{20}{*}{ May 2017} & \#20. \#17 AND \#18 AND \#19 & 37 \\
\hline & \#19. \#13 OR \#14 OR \#15 OR \#16 & 3,004 \\
\hline & \#19. \#13 OR \#14 OR \#15 OR \#16 & 64,034 \\
\hline & $\begin{array}{l}\# 17 . \# 1 \text { OR \#2 OR \#3 OR \#4 OR \#5 } \\
\text { OR \#6 }\end{array}$ & $3,512,600$ \\
\hline & \#16. 'plr' & 2,678 \\
\hline & \#15. 'platelet-to-lymphocyte ratio' & 766 \\
\hline & \#14. 'platelet to lymphocyte ratio' & 766 \\
\hline & \#13. 'platelet lymphocyte ratio'/exp & 1,245 \\
\hline & \#12. 'esophagus carcinoma' & 15,242 \\
\hline & \#11. 'esophageal carcinoma' & 7,771 \\
\hline & \#10. 'esophageal cancer' & 24,793 \\
\hline & \#9. 'esophagus neoplasm' & 17 \\
\hline & \#8. 'esophageal neoplasm' & 421 \\
\hline & \#7. 'esophagus cancer'/exp & 57,252 \\
\hline & \#6. 'mortality' & $1,073,552$ \\
\hline & \#5. 'survival' & $1,391,365$ \\
\hline & \#4. 'outcome' & $2,054,741$ \\
\hline & \#3. 'prognostic' & 359,252 \\
\hline & \#2. 'prognoses' & 9,581 \\
\hline & \#1. 'prognosis'/exp & 605,644 \\
\hline
\end{tabular}

\begin{tabular}{|c|c|c|}
\hline $\begin{array}{l}\text { Search } \\
\text { format }\end{array}$ & Results & Combine \\
\hline \multirow[t]{2}{*}{ \#4 } & 50 & \#3 AND \#2 AND \#1 \\
\hline & & $\begin{array}{l}\text { Index }=\text { SCI-EXPANDED, SSCI time } \\
\text { span }=1992-2017\end{array}$ \\
\hline \multirow[t]{2}{*}{ \#3 } & 2,777 & $\begin{array}{l}\text { Term: (platelet lymphocyte ratio) OR term: } \\
\text { (platelet to lymphocyte ratio) OR term: } \\
\text { (platelet-to-lymphocyte ratio) OR term: (PLR) }\end{array}$ \\
\hline & & $\begin{array}{l}\text { Index=SCI-EXPANDED, SSCI time span=all } \\
\text { years }\end{array}$ \\
\hline \multirow[t]{2}{*}{ \#2 } & 42,988 & $\begin{array}{l}\text { Term: (Esophageal Neoplasm) OR term: } \\
\text { (Esophagus Neoplasm) OR term: } \\
\text { (Esophagus Cancer) OR term: (Esophageal } \\
\text { Cancer) OR term: (esophageal carcinoma) } \\
\text { OR term: (Esophagus carcinoma) }\end{array}$ \\
\hline & & $\begin{array}{l}\text { Index=SCI-EXPANDED, SSCI time span=all } \\
\text { years }\end{array}$ \\
\hline \multirow[t]{2}{*}{$\# 1$} & $2,864,518$ & $\begin{array}{l}\text { Term: (Prognosis) OR term: (Prognoses) OR } \\
\text { term: (prognostic) OR term: (outcome) OR } \\
\text { term: (survival) OR term: (mortality) }\end{array}$ \\
\hline & & $\begin{array}{l}\text { Index=SCI-EXPANDED, SSCI time span=all } \\
\text { years }\end{array}$ \\
\hline
\end{tabular}

